\title{
Plasmonic nanoparticle based nanobiosensors and nanophotodetectors
}

Neval A. Cinel Serkan Bütün Ekmel Özbay 


\title{
Plasmonic nanoparticle based nanobiosensors and nanophotodetectors
}

\author{
Neval A. Cinel ${ }^{1, ~}$, Serkan Bütün ${ }^{2}$, Ekmel Özbay ${ }^{1,3}$ \\ ${ }^{1}$ Nanotechnology Research Center, Department of Electrical and Electronics Engineering, Bilkent University, Bilkent, \\ 06800 Ankara, Turkey \\ ${ }^{2}$ Technological Institute, Electrical Engineering and Computer Science Department, Northwestern University, Evanston, \\ IL 60208, U.S.A. \\ ${ }^{3}$ Nanotechnology Research Center, Department of Physics, Bilkent University, Bilkent, 06800 Ankara, Turkey \\ nyilmaz@ee.bilkent.edu.tr
}

\begin{abstract}
Plasmonics mainly deals with light-matter interactions in metallic nanostructures. It has gathered interest since its discovery due to the benefits it provides when compared with photonics and electronics. It owes its popularity to the tremendous number of applications it serves for. In this paper, we review how plasmonic nanoparticles can be utilized in applications such as localized surface plasmon resonance based biosensing and enhancing performance of photodetectors.
\end{abstract}

Keywords: plasmonics, biosensor, LSPR, MSM, photodetector.

\section{INTRODUCTION}

Plasmonics can be utilized in various applications such as increasing resolution in lithography, microscopy, enhancing surface enhanced Raman scattering [1,2], biosensing [3-5] and enhancing light trapping properties of thin film solar cells [6-9]. In this review, we firstly report the use of plasmonic silver nano-disks for localized surface plasmon resonance (LSPR) based biosensing. Then, we report how a periodic aluminum nano-dot array whose resonance is tuned for UV wavelengths can increase the detector responsivity in an MSM UV photodetector. Finally, the use of aluminum nano-grating structures which work as nano-antennas for light absorption enhancement in MSM photodetectors based on $\mathrm{GaN}$ is demonstrated.

\section{LABEL FREE LSPR BIOSENSORS}

Rapid, label-free, sensitive and real-time biosensing is vital especially in medical diagnostics, environmental monitoring and prevention of bio-terrorism. LSPR biosensors satisfy all these requirements with low cost and usage simplicity. The sensing mechanism of LSPR biosensors is based on the detection of changes in refractive index of the surrounding environment caused by the binding of the analyte to be detected to the sensor surface functionalized for detection [10]. The LSPR resonance wavelength is highly dependent on the nano-particles' size, size distribution and shape [11]. Therefore lithographic methods that end up with better uniformity and resolution offer a good alternative for fabrication.

In our study, the silver nanodisks on transparent sapphire substrate prepared with E-Beam Lithography (EBL) serve as a refractive index sensor [12]. The specificity of the sensor surface is maintained by the surface functionalization steps which include the formation of a self assembled monolayer and a biotinylation step as shown in Figure 1a. The biotin-avidin bioassay used for the verification of the sensor structure is known for one of the most strong non-covalent bonds that isn't affected by environmental extremes and hence preferred. 
Concentration and time dependency are two important features of biosensors. The response of the sensor was recorded at different analyte concentrations. Results showed that the sensor responds to changes in concentration at the $1 \mathrm{nM}-100 \mathrm{nM}$ range with a sigmoidal fitting which is typical of biosensing experiments as shown in Figure 1b [13-15]. Another experiment was conducted by recording the LSPR shifts in the first three hours for studying the time dependency. The time response of the sensor gets saturated within the first thirty minutes of the sensor which indicates opportunity of rapid detection as shown at the inset of Figure $1 \mathrm{~b}$.

A refractive index sensitivity of $354 \mathrm{RIU} / \mathrm{nm}$ is calculated for the sensor by using the wavelength shifts observed after application of two different refractive index materials, namely alcohol and water. This value is one of the high values reported in the literature for this type of sensors.

(a)

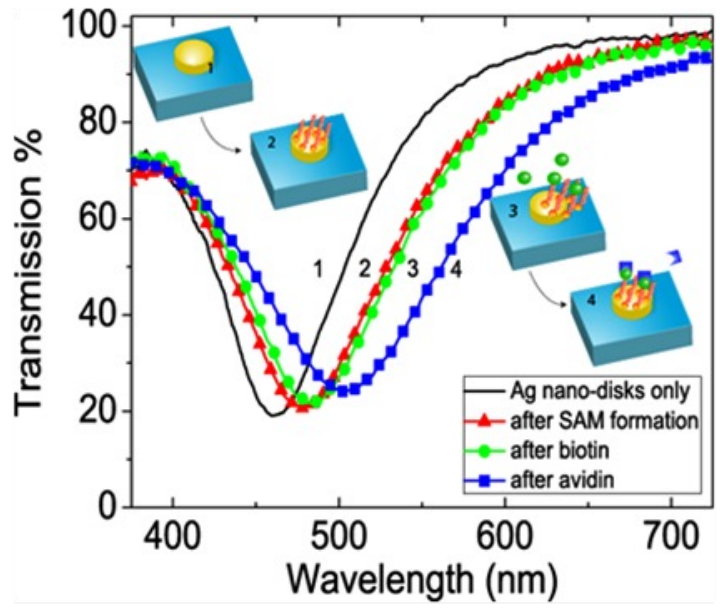

(b)

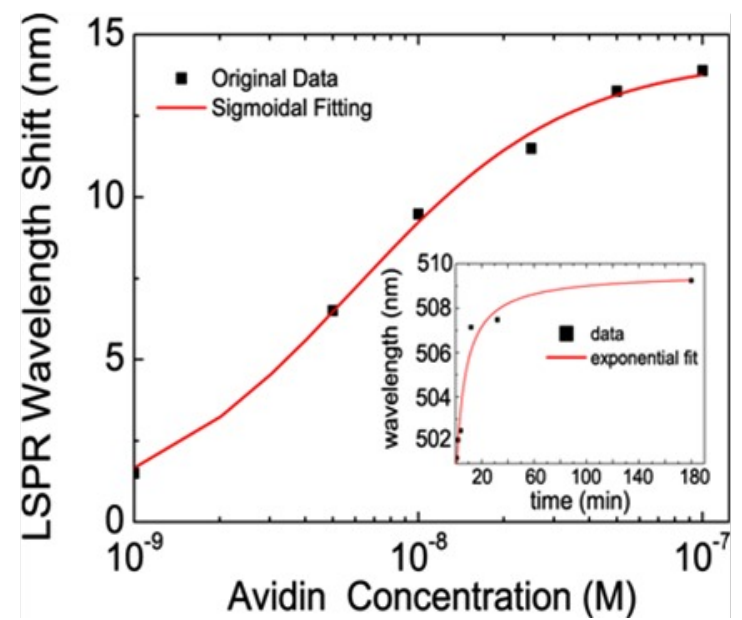

Fig. 1: (a) Transmission measurements after every surface functionalization step, respective surface functionalization steps are shown at the inset (b) concentration and time dependency (shown at the inset) of the LSPR sensor

A preliminary trial to use the same sensor structure for the detection of E-Coli bacteria has also been tried. The surface functionalization step was modified for this purpose. Biotin conjugated antibody of specific bacteria is immobilized onto the sensor in place of biotin, which could then be used to detect the presence of the antigen. This idea can be applied to other types of bacteria using similar functionalization methods. A consistent shift after application of bacteria is considered to imply the presence of the bacteria.

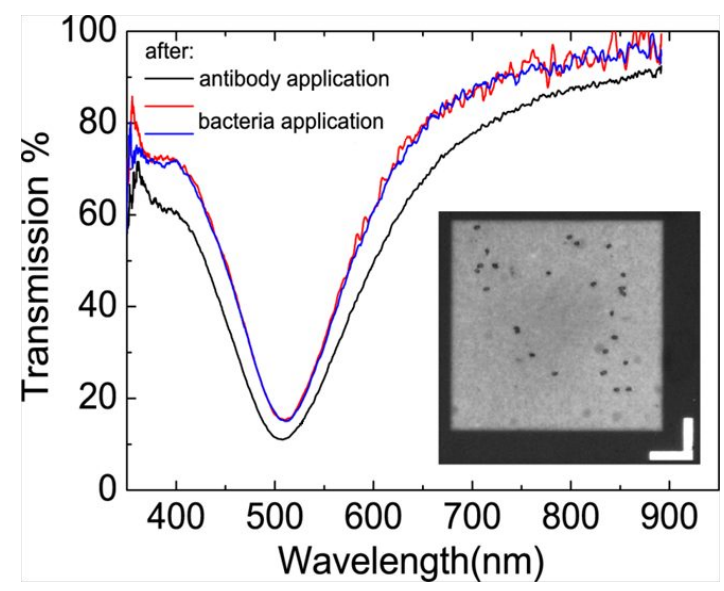

Fig. 2: (a) Transmission measurements after application of bacteria, the sensor surface under electron microscope is shown at the inset. The dark cylindrical rods are the bacteria, the sensor surface is $50 \times 50 \mu \mathrm{m}$. 


\section{LSPR ENHANCED MSM UV PHOTODETECTORS}

UV detectors find area of use in several civil and military applications such as space-to-space communications, ozone layer monitoring, early missile plume detection. $\mathrm{GaN}$ is one of the most favorable materials used in UV detectors owing to their high saturation electron drift velocity, radiation hardness, resistance to high temperatures and durability for extreme conditions. In this study, LSPR enhanced MSM photodetectors on GaN active layer operating at UV regime are designed [16]. The design consists of EBL fabricated Al nano-particles inserted between the Schottky contacts of a conventional MSM photodetector residing on MOCVD grown semi-insulating GaN. A schematic drawing of the fabricated device is shown in Figure 3a.
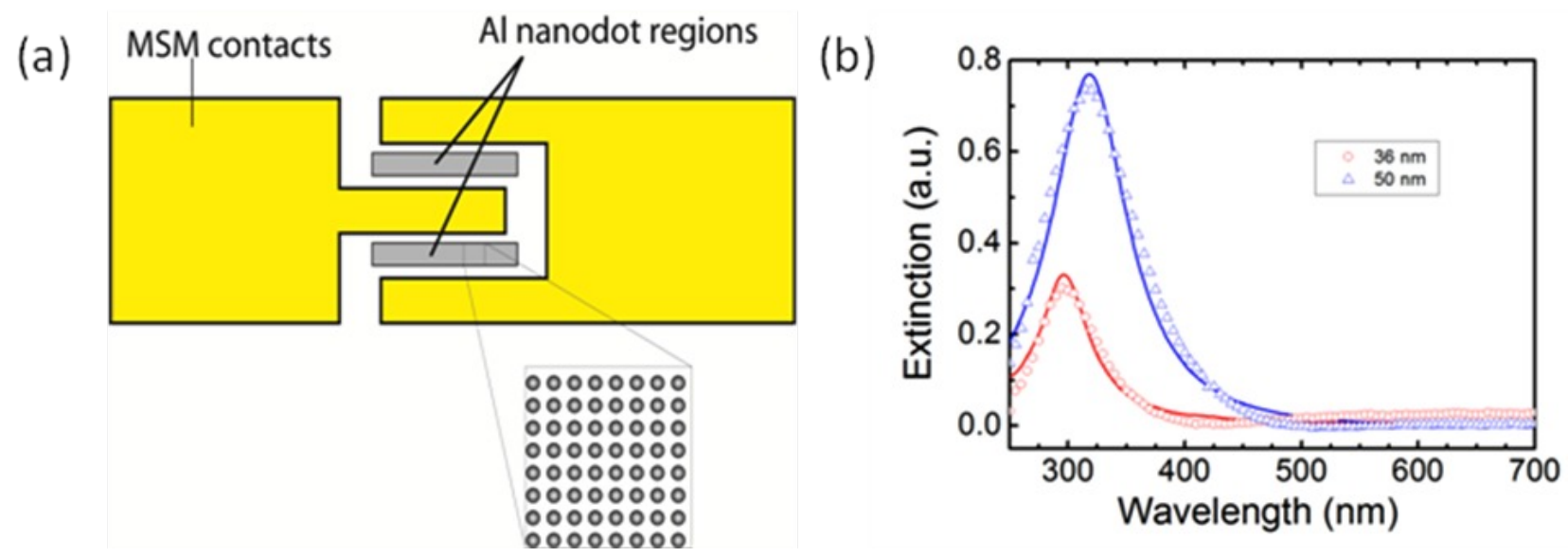

Fig. 3: (a) Conceptual drawing of the LSPR enhanced MSM photodetector. (b) The extinction spectra of the Al nano particles. Dots represent the measurements whereas lines correspond to FDTD simulations.

The motivation of this study was to tune the resonance of the nano-dots to UV wavelengths where GaN has high absorption to enhance the device responsivity. Al was chosen since it supports the surface plasmons at the UV regime. The optical characterization of the $\mathrm{Al}$ nano-dots fabricated on sapphire substrate was done through transmission measurements and FDTD simulations as indicated in Figure 3b. A well-established resonance around $300 \mathrm{~nm}$ was observed which is expected to shift toward longer wavelengths when same sized nano-dots are fabricated on GaN due to the higher refractive index of GaN. The resultant structure was compared to a conventional MSM photodetector on the same substrate without Al nano-dots.

Photodetector characterization requires the estimation of spectral responsivity. Responsivity is the ratio of generated photocurrent to the incident optical power and measured in amps per watt (A/W). A custom set-up was used to measure the generated photocurrent [16]. To determine the incident optical power we used a NIST-traceable, calibrated Si photodetector with known spectral responsivity. The measured responsivity was used in the determination of the quantum efficiency using the relation: $R=\eta \cdot(\lambda / 1.24)$, where $R$ is responsivity, $\eta$ is the quantum efficiency and $\lambda$ is the wavelength. The calculated quantum efficiencies are plotted in Figure 4a.

The enhancement in quantum efficiency is depicted in Figure 4b. About 50\% enhancement was observed in the presence of plasmonic nanoparticles when compared with the control sample without nanoparticles. The enhancement at the resonance wavelength around $340 \mathrm{~nm}$ is attributed to the strongly localized electromagnetic field of the Al nano-dots at their interface with the $\mathrm{GaN}$ active layer. 
(a)

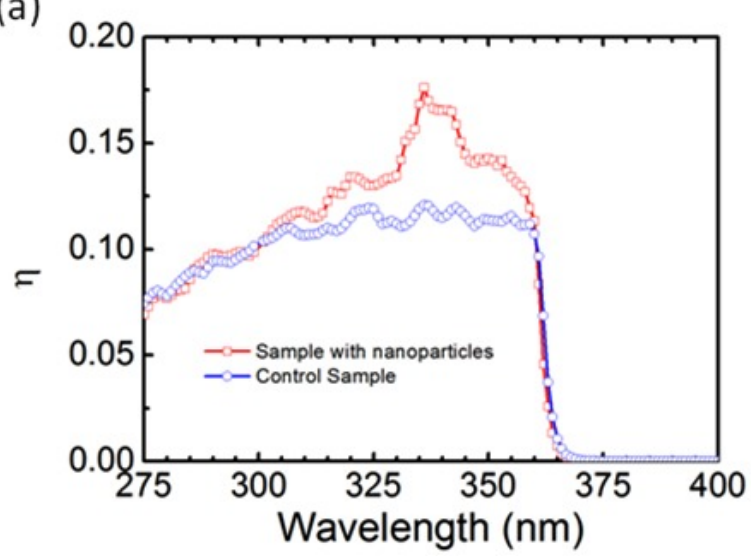

(b)

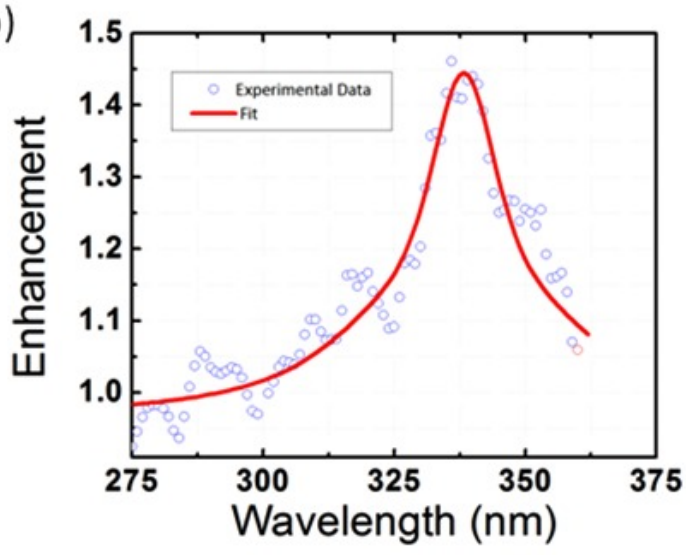

Fig 4. : (a) Spectral quantum efficiency measurement of LSPR enhanced photodetector along with the control sample (b) Enhancement of the quantum efficiency with insertion of Al nano-particles.

\section{NANO-ANTENNA COUPLED UV PHOTODETECTORS}

Solid-state devices incorporated with nano-structures can be successfully used in integrated circuits for better light integration performance [17]. Such devices are reported for the near-IR to visible region but studies for the UV region are still immature. In this study, a UV nano-antenna integrated metal semiconductor metal (MSM) photodetector based on $\mathrm{GaN}$ is implemented [18]. Our motivation was collecting more photons with the help of the Al grating nano-antenna fabricated on top of the photodetector with a subwavelength aperture. The light absorption enhancement is expected since more optical power would be captured and delivered to the absorbing active regions of the device.

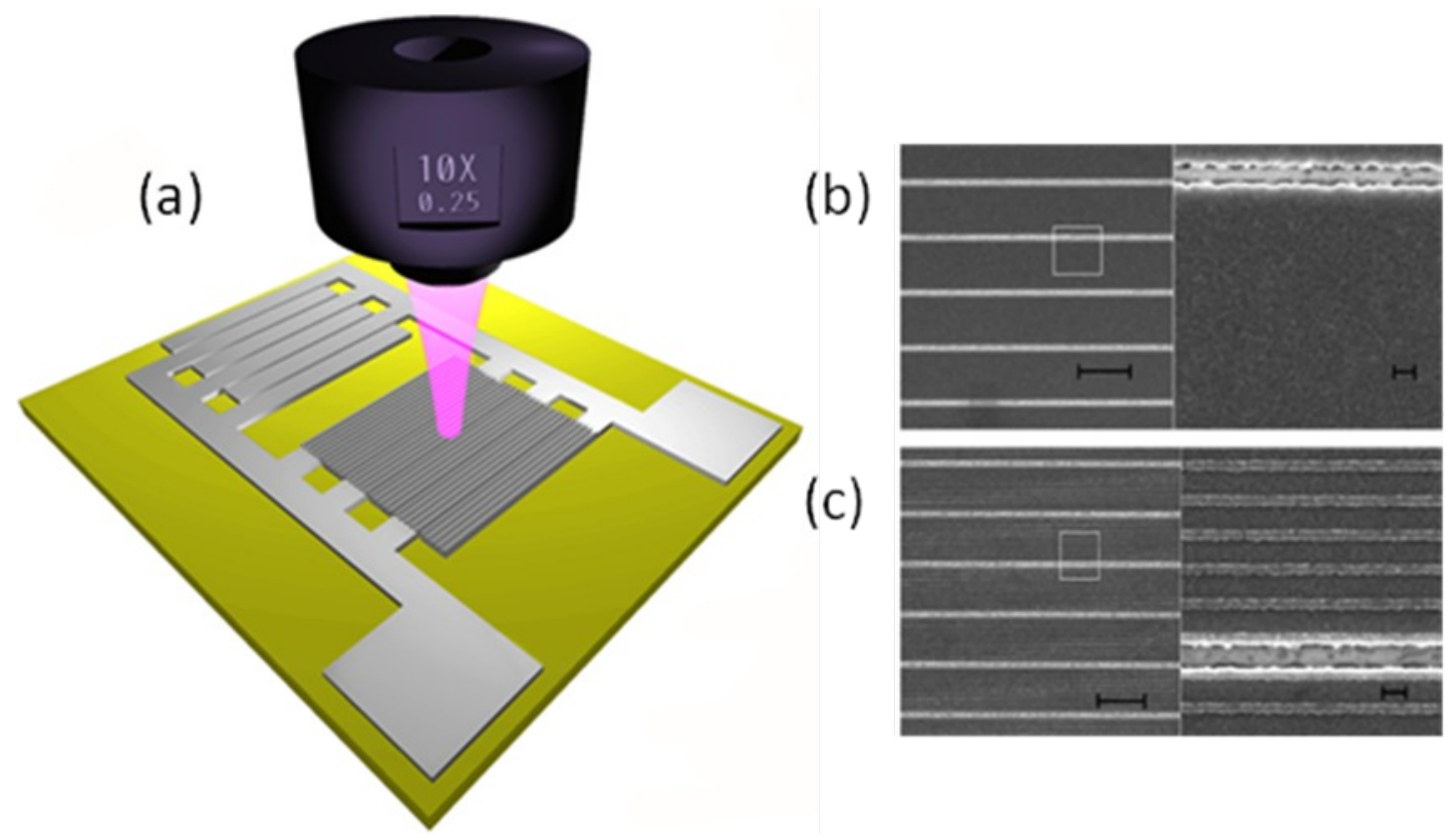

Fig. 5. : (a) Conceptual drawing of the photodetector structure. SEM images of the MSM contacts (b) without grating (c) with grating. The longer scale bar corresponds to $3 \mu \mathrm{m}$ and the shorter scale bar corresponds to $200 \mathrm{~nm}$. 
The conceptual drawing of the photodetector structure and the scanning electron microscopy (SEM) images are shown in Figure 5. All fabrication steps were performed by EBL. The MSM contact width was chosen as $3 \mu m$ to be below the propagation length calculated as $4.2 \mu \mathrm{m}$ for the $320 \mathrm{~nm}$ UV excitation so that waveguide modes of the slits could be excited via SPPs. The grating period was selected as $314 \mathrm{~nm}$ for phase matching of the surface plasmon polaritons with the excitation light and duty cycle was $1 / 3$.

The optical characterization and optimization of the nano-structures was done through the reflection measurements from samples with various grating periods on sapphire substrate with all the other physical parameters kept constant. Reflections were measured by ellipsometric measurements at the $70^{\circ}$ measurement angle. Samples without gratings are also prepared for the reference measurements. The observed resonant dips are due to coupling to SPPs, and they are red shifted as the grating period increases. The position of the dips are in accordance with the values calculated using the dispersion relation [18].
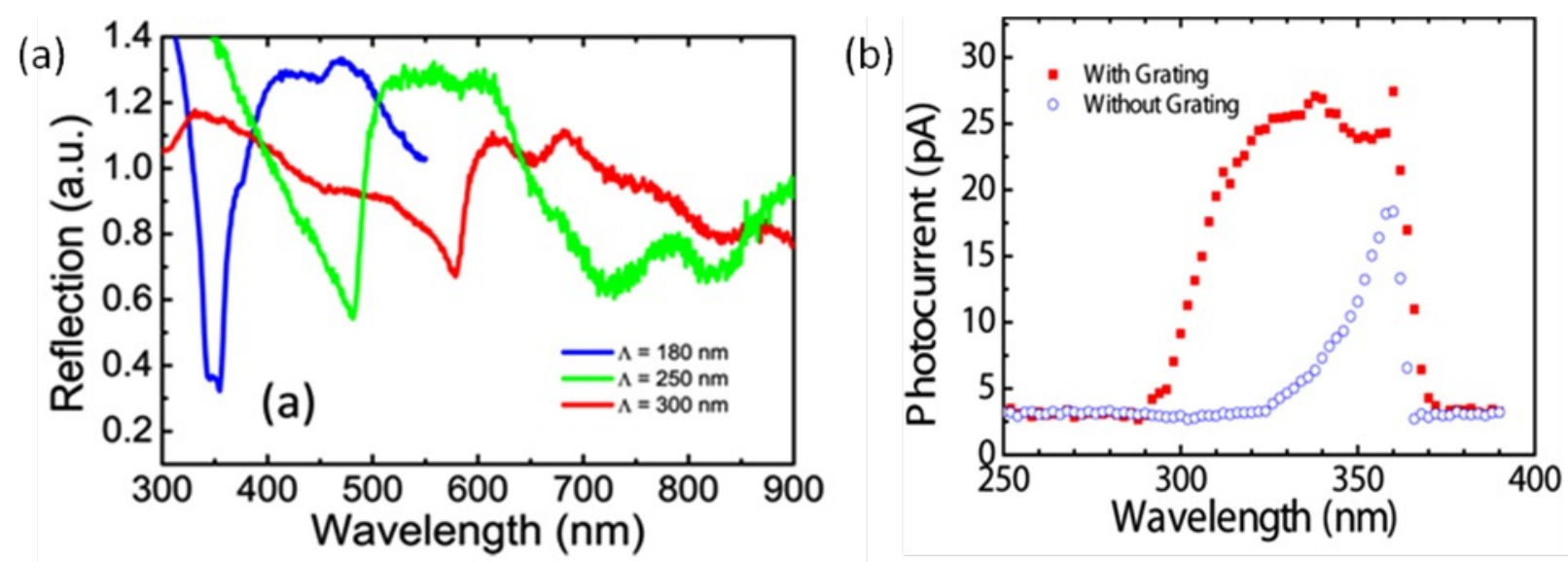

Fig. 6. : (a) Reflectance measurements of the Al gratings on sapphire with different periods. (b) Photocurrent measurements with and without gratings.

The photocurrent measurements were done by a synchronous detection scheme. The measured photocurrent data is shown compared in Fig 6 (b). At zero volt bias, the peak photocurrent value for the samples with grating is measured as $24.5 \mathrm{pA}$, whereas it is $3.4 \mathrm{pA}$ for the no grating site. These values correspond to the responsivities of 1.7 $m A / W$ and $0.2 m A / W$, respectively.

The FDTD simulations were done to depict the enhancement in absorption at the resonant and off-resonant cases. The total absorbed light intensity was calculated by the integration of the E-field intensity under the slits with and without the gratings for both cases. Fig. 7(a) and (b) compares the total amount of absorbed light at resonant (340 $\mathrm{nm})$ and off-resonant $(280 \mathrm{~nm})$ wavelengths, respectively. 

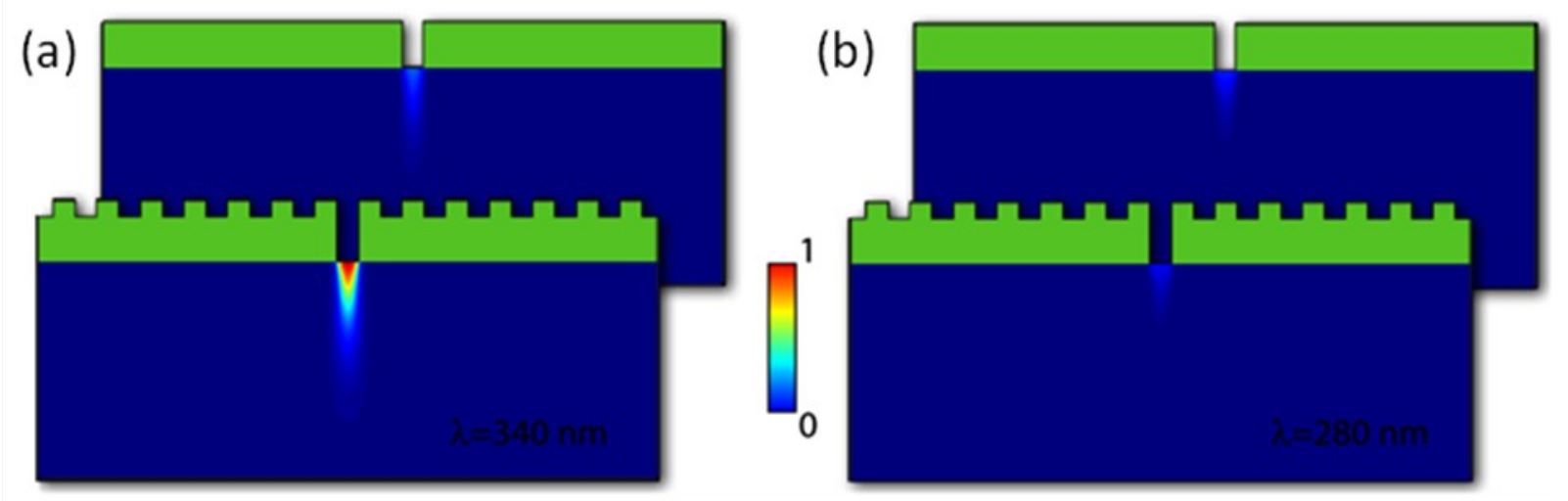

Fig. 7. : The overall normalized E-field intensity under the slits with and without the gratings at (a) a resonant and (b) an offresonant excitation.

In conclusion, we have designed an MSM detector with a sub-wavelength aperture surrounded by an Al nanostructured metal grating and compared to a conventional MSM photodetector employing only a sub-wavelength aperture. The gratings that act as nano-antennas collecting the optical signal into the absorbing active regions of the device leading to more than eight fold photocurrent enhancement.

\section{CONCLUSION}

Plasmonic nanoparticles have a wide variety of applications. In this paper, we review their utilization in biological sensing and photo-detection. In the first part, EBL fabricated plasmonic nano-disks functioning as LSPR based refractive index sensors were implemented. In the second part, the integration of nano structures with MSM photodetectors were shown to increase the coupling of more light into the device and thereby increase the responsivity and photocurrent enhancement.

\section{ACKNOWLEDGMENT}

This work is supported by the projects DPT-HAMIT, ESF-EPIGRAT, EU-N4E, NATO-SET-181 and TUBITAK under Project Nos., 107A004, 107A012, 109E301. We also acknowledge partial support from the Turkish Academy of Sciences.

\section{References}

[1] M. Kahl, E. Voges, S. Kostrewa, C. Viets and W. Hill, "Periodically structured metallic substrates for SERS," Sensor Actuat B-Chem. 51, 285 (1998).

[2] J. B. Jackson and N. J. Halas, "Surface-enhanced Raman scattering on tunable plasmonic nanoparticle substrates," Proceedings of the National Academy of Sciences 101, 17930 (2004).

[3] N. A. Cinel, S. Bütün and E. Özbay, "Electron beam lithography designed silver nano-disks used as label free nanobiosensors based on localized surface plasmon resonance," Opt. Express 20, 2587 (2012).

[4] J. N. Anker, W. P. Hall, O. Lyandres, N. C. Shah, J. Zhao and R. P. Van Duyne, "Biosensing with plasmonic nanosensors," Nature Materials 7, 442 (2008).

[5] J. Zhao, X. Zhang, C. R. Yonzon, A. J. Haes and R. P. Van Duyne, "Localized surface plasmon resonance biosensors," Nanomedicine 1, 219 (2006).

[6] Y. A. Akimov and W. Koh, "Optimization of light-trapping in thin-film solar cells enhanced with plasmonic nanoparticles," Photonics Global Conference, pp. 1-5 (2010),

[7] H. A. Atwater and A. Polman, "Plasmonics for improved photovoltaic devices," Nature Materials 9, 205 (2010). 
[8] Y. A. Akimov and W. S. Koh, "Design of plasmonic nanoparticles for efficient subwavelength light trapping in thin-film solar cells," Plasmonics 6, 155 (2011).

[9] Z. Ouyang, X. Zhao, S. Varlamov, Y. Tao, J. Wong and S. Pillai, "Nanoparticle enhanced light trapping in thin film silicon solar cells," Progress in Photovoltaics: Research and Applications 19, 917 (2011).

[10] K. A. Willets and R. P. Van Duyne, "Localized surface plasmon resonance spectroscopy and sensing," Annu. Rev. Phys. Chem. 58, 267 (2007).

[11] E. Hutter and J. H. Fendler, "Exploitation of localized surface plasmon resonance," Advanced Materials 16, 1685 (2004).

[12] N. A. Cinel, S. Bütün and E. Özbay, "Electron beam lithography designed silver nano-disks used as label free nanobiosensors based on localized surface plasmon resonance," Optics Express 20, 2587 (2012).

[13] T. Arai, P. K. R. Kumar, C. Rockstuhl, K. Awazu and J. Tominaga, "An optical biosensor based on localized surface plasmon resonance of silver nanostructured films," Journal of Optics A: Pure and Applied Optics 9, 699 (2007).

[14] A. J. Haes, W. P. Hall, L. Chang, W. L. Klein and R. P. Van Duyne, "A localized surface plasmon resonance biosensor: First steps toward an assay for Alzheimer's disease," Nano Lett. 4, 1029 (2004).

[15] J. C. Riboh, A. J. Haes, A. D. McFarland, C. R. Yonzon and R. P. Van Duyne, "A nanoscale optical biosensor: Real-time immunoassay in physiological buffer enabled by improved nanoparticle adhesion," J. Phy. Chem. B. 107, 1772 (2003).

[16] S. Butun, N. A. Cinel and E. Ozbay, "LSPR enhanced MSM UV photodetectors," Nanotechnology 23, 444010 (2012).

[17] E. Ozbay, "Plasmonics: merging photonics and electronics at nanoscale dimensions," Science 311, 189 (2006).

[18] S. Butun, N. A. Cinel and E. Ozbay, "Nano-antenna coupled UV subwavelength photodetectors based on GaN," Opt. Express 20, 2649 (2012). 